\title{
LMI Solution to Gain-constrained Robust Deadbeat Pole Assignment
}

\author{
Hei Ka Tam James Lam* \\ Department of Mechanical Engineering \\ University of Hong Kong \\ Pokfulam Road, HONG KONG
}

\begin{abstract}
A novel optimization approach to robust deadbeat control is proposed. The design problem is cast into a convex programming task in which a special measure of closed-loop eigenvalue sensitivity is minimized. Advantages of the proposed method include: (1) Global optimality is guaranteed when the solution set is nonempty; (2) Constraints on the feedback gain can be catered naturally; (3) Minimum-gain deadbeat control design can be readily treated.
\end{abstract}

\section{Introduction}

Consider a linear time-invariant MIMO discrete time system

$$
x_{t+1}=A x_{t}+B u_{t} \quad, \quad t=0,1,2, \ldots
$$

where $A \in \mathbb{R}^{n \times n}, B \in \mathbb{R}^{n \times q}, x_{t} \in \mathbb{R}^{n \times 1}, u_{t} \in \mathbb{R}^{q \times 1}$. Assume $B$ is of full column rank while $(A, B)$ is reachable with reachability indices $k=k_{1} \geq k_{2} \geq \ldots \geq k_{q}$. By applying a time-invariant state-feedback law

$$
u_{t}=K x_{t}
$$

there results the closed-loop system given by

$$
x_{t+1}=(A+B K) x_{t}
$$

The reachability of $(A, B)$ implies that the spectrum of the closed-loop state matrix $M \triangleq A+B K$ can be assigned to any arbitrary set of self-conjugate complex numbers by proper choice of the feedback gain matrix $K \in \mathbb{R}^{q \times n}$. It is often desirable to assign the set of closed-loop poles at the origin of the complex plane. In such case, the closed-loop system exhibits deadbeat characteristics, with which zero-input response of the system dies down to zero in finite time steps. Furthermore, in order for a system under Minimumtime Deadbeat Control ( $M T D C$ ), that is, the regulation time to drive every initial state of the closed-loop system to the origin is made shortest, $M$ is made similar to the block-diagonal matrix

$$
J=\operatorname{diag}\left(J_{1}, J_{2}, \ldots, J_{q}\right)
$$

where $J_{p}$ is a Jordan block of dimension $k_{p}$ (for $k_{p}=0$, $J_{p}$ does not appear in $J$ ).

* Supported by F[KU CRCG Grant

$0-7803-4530-4 / 93 \$ 10.00$ (c) 1998 AACC
By observing that $M$ is nilpotent with order of nilpotence equals $k\left(M^{k}=0\right)$ and generally has a nontrivial Jordan structure, it is especially susceptible to spectral variation as a result of perturbation. As the feedback gain to achieve pole assignment for multi-input systems is in general non-unique, it is particularly meaningful for the non-uniqueness to be exploited in searching for a more robust eigenstructure.

\section{Affine Parametrization of MTDC Feedback Gain}

The idea of explicitly parametrizing the class of deadbeat regulators proposed by Funahashi et al. [2] is borrowed. Initially, $(A, B)$ is transformed via $S \in$ $\mathbb{R}^{n \times n}$ into the Luenberger canonical form

$$
y_{t+1}=S A S^{-1} y_{t}+S B u_{t} \quad, \quad y_{t}=S x_{t}
$$

where

$$
S A S^{-1}=J+E \hat{A} \quad, \quad S B=E \hat{B}
$$

in which

$$
\begin{aligned}
E & =\text { block diag }\left(\mathbf{e}_{1}, \mathbf{e}_{2}, \ldots, \mathbf{e}_{q}\right) \in \mathbb{R}^{n \times q} \\
\mathbf{e}_{i} & =(0 \ldots 01)^{T} \in \mathbb{R}^{k_{i} \times 1}
\end{aligned}
$$

$\hat{A} \in \mathbb{R}^{q \times n}$ and $\hat{B} \in \mathbb{R}^{q \times q}$ are determined uniquely by $(A, B)$. The transformation (6) results in the closedloop state matrix being transformed into

$$
S(A+B K) S^{-1}=J+E \hat{K}
$$

where

$$
\hat{K}=\hat{A}+\hat{B} K S^{-1} \in \mathbb{R}^{q \times n}
$$

From Funahashi et al. [2] , it is not difficult to observe that $\hat{K}$ can be expressed as

$$
\hat{K}=\sum_{r=2}^{q} \sum_{s=1}^{r-1} \sum_{v=k_{r}+1}^{k_{s}} \gamma_{r, v+\sigma(s)} G_{r, v+\sigma(s)}
$$

where $\sigma(s)=\sum_{p=1}^{s-1} k_{p}$ in which $G_{r, v+\sigma(s)}$ denotes a matrix in $\mathbb{R}^{q \times n}$ whose elements equal zero except the $(r, v+\sigma(s))$ element equal to one. It follows from (7) and (8) that

$$
K(\mathbf{w})=K_{0}+\sum_{i=1}^{N} w_{i} K_{i} \quad, \quad \mathbf{w}=\left(w_{1} \ldots w_{N}\right)^{T}
$$


where

$$
\begin{aligned}
K_{0} & =-(\hat{B})^{-1} \hat{A} S \\
K_{i} & =(\hat{B})^{-1} G_{r, v+\sigma(s)} S \quad, \quad w_{i}=\gamma_{r, v+\sigma(s)}(10)
\end{aligned}
$$

with the indices $r, s, v$, and $i$ being related by

$$
i=\sum_{j=2}^{r-1} \sum_{l=1}^{j-1}\left(k_{l}-k_{j}\right)+(v+\sigma(s))-s k_{r}
$$

$N=n q-\sum_{p=1}^{q}(2 p-1) k_{p}$ is the minimum number of independent free parameters $w_{i}$ in parametrizing $K$.

\section{Measure of Robustness}

Recently, Lam et al. [3] have shown that the spectral norm of $M$ serves as a special measure of eigenvalue sensitivity for deadbeat control systems. In the following, $\|M\|_{2}$ and $\|M\|_{\max }$ denotes respectively the spectral norm and the maximum norm of $M$ ( maximum absolute value of the entries in $M$ ).

Theorem 1 Suppose $M$ is subjected to a perturbation $\Delta$ where $\lambda$ is an eigenvalue of $M+\Delta$, then we have: ( $I$ )

$$
\begin{aligned}
& |\lambda| \leq \max _{i=0, \ldots, k-1}\left\{\left(k\left\|M^{i} \Delta\right\|_{2}\right)^{\frac{1}{i+1}}\right\}, \\
& |\lambda| \leq \max _{i=0, \ldots, k-1}\left\{\left(k\left\|M^{i}\right\|_{2}\|\Delta\|_{2}\right)^{\frac{1}{i+1}}\right\}, \\
& |\lambda| \leq \max _{i=0, \ldots, k-1}\left\{\left(k\|M\|_{2}^{i}\|\Delta\|_{2}\right)^{\frac{1}{i+1}}\right\} ;
\end{aligned}
$$

and (II)

$$
\begin{aligned}
& |\lambda| \leq(1+\epsilon)\left\|M^{k-1}\right\|_{2}^{\frac{1}{k}}\|\Delta\|_{2}^{\frac{1}{k}} \leq(1+\epsilon)\|M\|_{2}^{\frac{k-1}{k}}\|\Delta\|_{2}^{\frac{1}{k}} \\
& \text { where } \epsilon=\mathcal{O}(|\lambda|) \rightarrow 0 \text { as }\|\Delta\|_{2} \rightarrow 0 .
\end{aligned}
$$

It turns out that variations on the perturbed closedloop eigenvalues would be small if $\|M\|_{2}$ is minimized.

\section{Robust Pole Assignment}

The Gain-constrained Robust Deadbeat Pole Assignment (GCRDPA) problem is now formulated as finding a state feedback gain $K$ of constrained size to assign the set of closed-loop poles into the origin of the $z$-plane and at the same time minimize the sensitivity of the closed-loop zero eigenvalues. The problem is cast into a constrained minimization task:

$$
\underset{\mathbf{w} \in \mathcal{P} \cap \mathcal{Q}}{\operatorname{minimize}} \phi(\mathbf{w})=\|A+B K(\mathbf{w})\|_{2}
$$

where

and

$$
\mathcal{P} \triangleq\left\{\mathbf{w} \mid\|K(\mathbf{w})\|_{2}<\alpha\right\}
$$

$$
\mathcal{Q} \triangleq\left\{\mathbf{w} \mid\|K(\mathbf{w})\|_{\max }<\beta\right\}
$$

Since $\phi(\mathbf{w})$ together with $\mathcal{P}, \mathcal{Q}$, and $\mathcal{P} \cap \mathcal{Q}$ are all convex in $\mathbf{w}$, (14) can be readily solved by state-ofthe-art convex optimization routines. Moreover, (14) can be recast into a Semidefinite Programming (SDP) task in which a functional $z$ is minimized subject to a set of Linear Matrix Inequality (LMI) constraints [1] :

$$
\underset{\mathbf{w}}{\operatorname{minimize}} z
$$

subject to

$$
\begin{aligned}
& \left(\begin{array}{cc}
z I_{n} & M(\mathbf{w}) \\
M(\mathbf{w})^{T} & z I_{n}
\end{array}\right)>0 \\
& \left(\begin{array}{cc}
\alpha I_{q} & K(\mathbf{w}) \\
K(\mathbf{w})^{T} & \alpha I_{n}
\end{array}\right)>0
\end{aligned}
$$

and

$$
-\beta \boldsymbol{\zeta}<\mathbf{h}+F \mathbf{w}<\beta \boldsymbol{\zeta}
$$

where

$F=\left(\begin{array}{llll}\operatorname{vec}\left(K_{1}\right) & \operatorname{vec}\left(K_{2}\right) & \cdots & \operatorname{vec}\left(K_{N}\right)\end{array}\right) \in \mathbb{R}^{(q \times n) \times N}$, $\mathbf{h}=\operatorname{vec}\left(K_{0}\right) \in \mathbb{R}^{(q \times n) \times \mathbf{1}}, \boldsymbol{\zeta}=(1 \ldots 1)^{T} \in \mathbb{R}^{(q \times n) \times 1}$, and vec(.) denotes the lexicographical ordering of the elements of a matrix. Notice that (20) is a pair of polyhedron constraints which can be represented as

$$
\operatorname{diag}(\beta \zeta-\mathbf{h}-F \mathbf{w})>0
$$

and

$$
\operatorname{diag}(\beta \zeta+\mathbf{h}+F \mathbf{w})>0
$$

It turns out that if (18), (19), and (20) are feasible ( i.e. $\mathcal{P} \cap \mathcal{Q} \neq \emptyset$ ), the solution produced by (17) will be globally optimal.

Remark 1 In (15) and (16), the greatest lower bound for $\alpha$ and $\beta$ to achieve deadbeat pole assignment can be computed using the following SDP settings.

To determine $\inf _{\mathbf{w}} \alpha$ :

$$
\underset{\mathbf{w}}{\operatorname{minimize}} \alpha
$$

subject to

$$
\left(\begin{array}{cc}
\alpha I_{q} & K(\mathbf{w}) \\
K(\mathbf{w})^{T} & \alpha I_{n}
\end{array}\right)>0
$$

To determine $\inf _{\mathrm{w}} \beta$ :

$$
\underset{\mathbf{w}}{\operatorname{minimize}} \beta
$$

subject to

and

$$
\operatorname{diag}(\beta \boldsymbol{\zeta}-\mathbf{h}-F \mathbf{w})>0
$$

$$
\operatorname{diag}(\beta \boldsymbol{\zeta}+\mathbf{h}+F \mathbf{w})>0
$$

Notice that (23) and (25) by themselves serve as computational procedures for solving the Minimum-gain Deadbeat Pole Assignment (MGDPA) problem.

\section{References}

[1] S. Boyd, L. E. Ghaoui, E. Feron, and V. Balakrishnan. Linear Matrix Inequalities in System and Control Theory, volume 15. SIAM Studies in Applied Mathematics, 1994.

[2] Y. Funahashi and M. Yamada. Explicit parameterization of state deadbeat controllers. I.E.E.E. Trans. Automatic Control, 37(10):1584-1588, 1992.

[3] J. Lam, H. K. Tso, and N. K. Tsing. Robust deadbeat regulation. Int. J. Control, 67:587-602, 1997. 